\title{
Predictors of Start of Different Antidepressants in Patient Charts Among Patients with Depression
}

\author{
Hyungjin Myra Kim, ScD; Kara Zivin, PhD; Hae Mi Choe, PharmD; Clare M. Stano, MA; \\ Dara Ganoczy, MPH; Heather Walters, MS; and Marcia Valenstein, MD
}

\begin{abstract}
BACKGROUND: In usual psychiatric care, antidepressant treatments are selected based on physician and patient preferences rather than being randomly allocated, resulting in spurious associations between these treatments and outcome studies.
\end{abstract}

OBJECTIVE: To identify factors recorded in electronic medical chart progress notes predictive of antidepressant selection among patients who had received a depression diagnosis.

METHODS: This retrospective study sample consisted of 556 randomly selected Veterans Health Administration patients diagnosed with depression from April 1, 1999, to September 30, 2004, stratified by the antidepressant agent, geographic region, gender, and year of depression cohort entry. Predictors were obtained from administrative data, and additional variables were abstracted from electronic medical chart notes in the year prior to the start of the antidepressant in 5 categories: clinical symptoms and diagnoses, substance use, life stressors, behavioral/ideation measures (e.g., suicide attempts), and treatments received. Multinomial logistic regression analysis was used to assess the predictors associated with different antidepressant prescribing, and adjusted relative risk ratios (RRR) were reported.

RESULTS: Of the administrative data-based variables, gender, age, illicit drug abuse or dependence, and number of psychiatric medications in the prior year were significantly associated with antidepressant selection. After adjusting for administrative data-based variables, sleep problems (relative risk ratio $[R R R]=2.47$ ) or marital issues $(R R R=2.64$ ) identified in the charts were significantly associated with prescribing mirtazapine rather than sertraline; however, no other chart-based variables showed a significant association or an association with a large magnitude.

CONCLUSIONS: Some chart data-based variables were predictive of antidepressant selection, but we neither found many nor found them highly predictive of antidepressant selection in patients treated for depression.

J Manag Care Spec Pharm. 2015;21(5):424-30

Copyright $\odot 2015$, Academy of Managed Care Pharmacy. All rights reserved.

\section{What is already known about this subject}

Patient characteristics are associated with antidepressant selection in patients treated for depression.

\section{What this study adds}

After adjusting for administrative data-based variables, we did not find chart-based variables to be highly predictive of antidepressant selection, particularly across SSRIs, in patients treated for depression.

Studies comparing outcomes associated with different antidepressant agents may not benefit greatly by further adjusting for variables obtained from medical records data beyond risk adjustment by administrative data-based variables.

T here is little empirical evidence to guide clinicians in selecting among the many antidepressants that are available, but in usual care, treatments are selected based on physician and patient preferences rather than being randomly allocated. ${ }^{1}$ For example, specific treatments may be preferentially prescribed to patients who have higher levels of suicidality or those with weight concerns, resulting in spurious associations between these treatments and outcomes such as suicides or cardiovascular events. ${ }^{2-5}$

Several variables, such as gender, age, ethnicity, weight, and mental health diagnoses, are easily ascertained by clinicians and can be obtained from health care administrative data. Affective states and behaviors such as suicidal attempts also clearly contribute to treatment selection, and standard medical documentation in electronic medical records from routine clinical visits may provide valuable information concerning various indications for selecting a particular antidepressant over another. Such chart data may be useful to clinicians, researchers, and health care systems for completing risk assessments to better adjust for selection biases.

The objective of this study was to examine factors available in the electronic medical record that are predictive of antidepressant selection, after adjusting for variables readily available in health system administrative data.

\section{Methods}

Retrospective chart abstraction was conducted for a sample of Veterans Health Administration (VHA) patients from the cohort of veterans with depression diagnoses who received care from April 1999 to September 2004. Entry into the depression cohort was defined by either 2 diagnoses of a depressive disorder or a depression diagnosis and an 
antidepressant prescription fill. Depression diagnoses were identified using the following International Classification of Diseases, Ninth Revision, Clinical Modification (ICD-9-CM) codes: 296.2x, 296.3x, 296.90, 296.99, 298.0, 300.4, 311, 293.83, 301.12 , 309.0, or 309.1. Patients were excluded if they received a diagnosis of bipolar I, schizophrenia, or schizoaffective disorder (about 15\%), since these diagnoses substantially change treatment approaches. More information about the cohort can be found in our previous study. ${ }^{6}$

Patients were randomly selected from the cohort for chart review, stratified by the newly initiating antidepressant agent, geographic region of the patient's VHA facility of most use, gender, and year of depression cohort entry. Seven different antidepressant agents most commonly prescribed at the VHA facilities during the study period were included: 4 selective serotonin reuptake inhibitors (SSRIs; citalopram, fluoxetine, paroxetine, and sertraline) and 3 non-SSRIs (bupropion, venlafaxine, and mirtazapine). Escitalopram was not in the VHA formulary during this study period. A "new" antidepressant start was defined as an antidepressant start that is preceded by at least 12 months of a clean period where no antidepressant prescription was filled. Since our interest was only to compare initially prescribed antidepressant agents, we considered neither the duration of initial prescription nor the presence of any subsequent antidepressant changes. Samples were chosen from only those with at least 1 outpatient visit in the year prior to the start of the antidepressant in order to allow medical records to be abstracted. This study was conducted with institutional review board approval from the Department of Veterans Affairs Health System.

Administrative data variables included demographic variables, mental health diagnoses, service connectedness (indicating disability from conditions occurring during or exacerbated by military service), Charlson Comorbidity Index, ${ }^{7}$ and numbers of VHA psychiatric hospitalizations, psychiatric inpatient days, and psychotropic medications. ${ }^{8}$ All comorbidities were defined based on data during the year prior to the index date.

We abstracted all medical record data for the year prior to and including the new start date of the antidepressant to construct various chart-based variables that might be captured in clinical notes but not in administrative data. Manual chart reviews were completed with the aid of a previously validated electronic medical record search engine (EMERSE) that highlights words in predefined search bundles. ${ }^{9}$ Further details on the search bundles, abstraction process, and chart-based variables can be found in our previous study. ${ }^{10}$

An extensive number of variables was abstracted from 5 categories: (1) clinical symptoms or diagnosis variables, (2) substance use variables, (3) life events or stressors, (4) behavioral/ideation measures indicating higher suicide risk (e.g., suicidal or aggressive behaviors), and (5) mental health or substance use treatments received. Briefly, clinical symptoms or diagnosis variables included those of anxiety, depression, mania, psychosis, impulsivity, pain, and sleep problems. Substance use variables included alcohol problem use/abuse/dependence, illicit drug use/dependence (excluding marijuana use, unless dependence was noted), and tobacco use as well as presence of prescription drug misuse based on notations that the patient took the prescription drugs recreationally, took prescription medications not prescribed to them, or took more than the prescribed amount. Life events or stressors included 12 items based on Axis IV of the Diagnostic and Statistical Manual of Mental Disorders: suicide of a family member, death of a family member, problems with primary support group, marital problem, social environment problem, difficulty with access to health care services, interaction with legal system/crime, other psychosocial and environmental problems, chronic illness/ pain, educational problems, occupational problems, and housing problems. Suicide risk-related behavioral measures included suicidal attempts, suicidal planning, suicidal ideation, suicidal thoughts, violent ideation, aggression, homicidal thoughts, type and availability of suicidal means, and access to guns..$^{11,12}$ Mental health or substance use treatments included participation in a homelessness intervention, VHA substance abuse treatment or alcoholics anonymous or self-help substance abuse treatment, and hospitalization considered due to psychological reasons by the provider. All measures were assessed based on all chart notations during the prior year and were dichotomized to "present" if assessed and present versus "not" if assessed and not present or if not mentioned.

\section{Data Analysis}

Distributions of chart-abstracted variables were compared across the 7 antidepressants. Because 7 antidepressant agents were considered, a multinomial logistic regression model was used to assess the independent relationship between various administrative data-based and chart-based variables and the choice of an antidepressant agent. We used sertraline as the referent medication to estimate the relative risk ratios (RRRs) associated with receiving 1 antidepressant treatment, each compared with sertraline, for various patient variables. Choosing sertraline as the referent medication meant that the comparisons will be relative to sertraline, but it does not make a difference in evaluating the associations between potential predictors and the choice of antidepressant agents. Because the goal was to assess if chart-based variables added more information beyond the variables available in administrative data, we identified significant administrative data-based variables first and then added chart-based variables while keeping in the model the administrative data-based variables identified to be significant. The sets of potential predictors were conceptually sequenced to represent unique contributions starting from demographics variables. Each set of chart-based predictors were sequentially added from mental health diagnoses and symptoms, life stressors, and behavioral or ideation measures to prior treatment-related variables in order to assess the 
Predictors of Start of Different Antidepressants in Patient Charts Among Patients with Depression

TABLE 1 Demographic Characteristics by the Newly Starting Antidepressants After at Least 12 Months of No Antidepressant Use in Randomly Selected Patients from April 1999 to September 2004 (N=556)

\begin{tabular}{|c|c|c|c|c|c|c|c|c|c|c|c|c|c|c|}
\hline \multirow[b]{4}{*}{ Characteristics } & \multicolumn{8}{|c|}{ SSRI } & \multicolumn{6}{|c|}{ Non-SSRI } \\
\hline & \multirow{2}{*}{\multicolumn{2}{|c|}{$\begin{array}{c}\text { Citalopram } \\
\mathrm{n}=78\end{array}$}} & \multirow{2}{*}{\multicolumn{2}{|c|}{$\begin{array}{c}\text { Fluoxetine } \\
\mathbf{n}=76\end{array}$}} & \multirow{2}{*}{\multicolumn{2}{|c|}{$\begin{array}{c}\text { Paroxetine } \\
\mathrm{n}=73\end{array}$}} & \multirow{2}{*}{\multicolumn{2}{|c|}{$\begin{array}{c}\text { Sertraline } \\
n=64\end{array}$}} & \multirow{2}{*}{\multicolumn{2}{|c|}{$\begin{array}{c}\text { Bupropion } \\
n=86\end{array}$}} & \multirow{2}{*}{\multicolumn{2}{|c|}{$\begin{array}{c}\text { Mirtazapine } \\
\mathbf{n}=98\end{array}$}} & \multirow{2}{*}{\multicolumn{2}{|c|}{$\begin{array}{c}\text { Venlafaxine } \\
n=81\end{array}$}} \\
\hline & & & & & & & & & & & & & & \\
\hline & $\%$ & (n) & $\%$ & (n) & $\%$ & (n) & $\%$ & (n) & $\%$ & (n) & $\%$ & (n) & $\%$ & (n) \\
\hline Male & 94.9 & (74) & 88.2 & (67) & 84.9 & (62) & 98.4 & (63) & 86.1 & (74) & 94.9 & (93) & 91.4 & (74) \\
\hline \multicolumn{15}{|l|}{ Age } \\
\hline$<50$ years & 26.9 & $(21)$ & 34.2 & (26) & 23.3 & (17) & 21.9 & (14) & 48.8 & $(42)$ & 32.7 & (32) & 28.4 & (23) \\
\hline $50-59$ years & 25.6 & (20) & 30.3 & (23) & 31.5 & (23) & 34.4 & (22) & 30.2 & (26) & 32.7 & (32) & 32.1 & (26) \\
\hline $60-69$ years & 19.2 & (15) & 19.7 & (15) & 21.9 & (16) & 20.3 & (13) & 16.3 & (14) & 13.3 & (13) & 16.1 & (13) \\
\hline$\geq 70$ years & 28.2 & (22) & 15.8 & (12) & 23.3 & (17) & 23.4 & (15) & 4.7 & (4) & 21.4 & (21) & 23.5 & (19) \\
\hline Mean (SD) & 59.4 & (14.4) & 55.7 & (13.0) & 59.1 & (13.3) & 59.1 & (13.4) & 50.6 & (11.9) & 56.3 & $(14.0)$ & 56.9 & (14.1) \\
\hline \multicolumn{15}{|l|}{ Race } \\
\hline White & 91.0 & $(71)$ & 82.9 & (63) & 76.7 & (56) & 82.8 & (53) & 82.6 & $(71)$ & 74.5 & (73) & 82.7 & (67) \\
\hline Black & 7.7 & (6) & 11.8 & (9) & 21.9 & (16) & 12.5 & (8) & 14.0 & (12) & 21.4 & (21) & 13.6 & (11) \\
\hline $\begin{array}{l}\text { Other/ } \\
\text { unknown }\end{array}$ & 1.3 & (1) & 5.3 & (4) & 1.4 & (1) & 4.7 & (3) & 3.5 & (3) & 4.1 & (4) & 3.7 & (3) \\
\hline Hispanic & 7.7 & (6) & 1.3 & (1) & 2.7 & (2) & 3.1 & (2) & 2.3 & (2) & 10.2 & (10) & 3.7 & (3) \\
\hline $\begin{array}{l}\text { Years since } \\
\text { depression } \\
\text { diagnosis, } \\
\text { mean (SD) }\end{array}$ & 0.2 & $(0.7)$ & 0.2 & $(0.5)$ & 0.2 & (0.6) & 0.2 & $(0.4)$ & 0.4 & (1.0) & 0.4 & $(0.7)$ & 0.4 & $(0.8)$ \\
\hline
\end{tabular}

Note: Distributions of demographic variables across all antidepressant agents are significant for male $(P=0.03)$, age $(P=0.03$, chi-square test; $P<0.001$, analysis of variance), Hispanic ethnicity $(P=0.05)$, and years since depression diagnosis $(P=0.02)$

$S D=$ standard deviation; $S S R I=$ selective serotonin reuptake inhibitor.

relationship between the potential predictors in each set and the choice of an antidepressant agent, while taking into account the association between the predictors in the previous set and antidepressant agents. At each model selection step, the variables in the set were selected using forward and backward stepwise regression in order to ensure stability of the selected predictors, while the variables selected from the previous sets were locked in the model. All analyses were conducted using Stata 13.0 (StataCorp College Station, TX), and an association was considered statistically significant at an alpha of 0.05.

\section{Results}

Table 1 shows the demographic variables by the 7 antidepressants. Unadjusted distributions of demographic variables across medication types were significant for male $(P=0.03)$, Hispanic ethnicity $(P=0.05)$, and age $(P=0.03)$. Of note, those who started bupropion were significantly younger than those who started other medications (50.6 years vs. 57.6 years, $P<0.0001$ ). Years since depression diagnosis was associated with medication types $(P=0.02)$, and in particular, patients on SSRIs had fewer years since depression diagnosis than those on medications other than SSRIs (0.19 vs. $0.42, P=0.0002)$. Table 2 shows the distribution of mental health diagnoses and other administrative data-based variables and antidepressant agents. Mental health diagnoses other than alcohol abuse/dependence and tobacco use diagnoses were not predictive of antidepres- sant selection, but severity measures (past psychiatric hospitalization and psychotropic medications) were significant predictors of selecting different antidepressant agents.

Table 3 shows the distribution of chart-based variables of mental health-related symptoms, substance use, stressful life events, suicidality, and mental health treatments received or considered by the 7 antidepressants. Sleep problems, tobacco use, and suicidal ideation or attempts were associated with antidepressant selection. The number of stressful life events was higher in patients prescribed mirtazapine than any other agent and was highest in citalopram among SSRIs. Of the stressful life events, presence of marital problems and social environment problems were again highest in mirtazapine and higher in citalopram and sertraline among SSRIs.

Table 4 shows the RRRs from the multinomial logistic regression model. Compared with sertraline, males were less likely to be prescribed paroxetine $(\mathrm{RRR}=0.11)$. Older patients were less likely to be prescribed fluoxetine $(R R R=0.97)$ and bupropion $(\mathrm{RRR}=0.94)$ compared with sertraline. Patients with illicit drug abuse or dependence were significantly less likely to be prescribed bupropion $(R R R=0.24)$ and mirtazapine $(R R R=0.22)$ than sertraline. Patients with more years since their first depression diagnosis tended not to be prescribed SSRIs, but the difference was not statistically significant. Although total number of prior psychiatric hospitalizations was not associated with the selection of different 
Predictors of Start of Different Antidepressants in Patient Charts Among Patients with Depression

TABLE 2 Administrative Data: Diagnoses and Utilization During the 12 Months Prior to Newly Starting Antidepressant

\begin{tabular}{|c|c|c|c|c|c|c|c|}
\hline \multirow[b]{3}{*}{ Characteristics } & \multicolumn{4}{|c|}{ SSRI } & \multicolumn{3}{|c|}{ Non-SSRI } \\
\hline & Citalopram & Fluoxetine & Paroxetine & Sertraline & Bupropion & Mirtazapine & Venlafaxine \\
\hline & $\mathrm{n}=78$ & $\mathrm{n}=76$ & $\mathrm{n}=73$ & $n=64$ & $\mathrm{n}=86$ & $\mathrm{n}=98$ & $\mathrm{n}=81$ \\
\hline Personality disorder (\%) & 3.9 & 2.6 & 2.7 & 0.0 & 1.2 & 1.0 & 4.9 \\
\hline Bipolar 2 (\%) & 1.3 & 0 & 0 & 0 & 2.3 & 2.0 & 1.2 \\
\hline Other anxiety disorder (\%) & 16.7 & 15.8 & 24.7 & 17.2 & 15.1 & 18.4 & 13.6 \\
\hline PTSD (\%) & 14.1 & 10.5 & 12.3 & 9.4 & 10.5 & 21.4 & 19.8 \\
\hline Service connected status (\%) & 25.6 & 25.0 & 23.3 & 23.4 & 37.2 & 31.6 & 32.1 \\
\hline Charlson index & $(1.4)$ & $0.8 \quad(1.1)$ & $0.8 \quad(1.3)$ & $1.0 \quad(2.1)$ & $1.0 \quad(1.8)$ & $1.0 \quad(2.0)$ & $0.8 \quad(1.5)$ \\
\hline Alcohol abuse/dependence (\%)a & 15.4 & 6.6 & 12.3 & 18.8 & 14.0 & 25.5 & 13.6 \\
\hline Illicit drug abuse/dependence (\%) & 7.7 & 6.6 & 9.6 & 17.2 & 7.0 & 15.3 & 12.4 \\
\hline Tobacco use diagnosis (\%) ${ }^{\mathrm{b}}$ & 15.4 & 10.5 & 15.1 & 17.2 & 37.2 & 12.2 & 16.1 \\
\hline Suicide attempt (\%)c & 1.3 & 0 & 0 & 0 & 0 & 0 & 1.2 \\
\hline \multicolumn{8}{|l|}{ Number of } \\
\hline Psychiatric hospitalizations ${ }^{a}$ & $0.1 \quad(0.3)$ & $0.01 \quad(0.1)$ & $0.1 \quad(0.4)$ & $0.2 \quad(0.4)$ & $0.1 \quad(0.3)$ & $0.3 \quad(0.7)$ & $0.1 \quad(0.5)$ \\
\hline Nonpsychiatric hospitalizations & $0.2 \quad(0.7)$ & $0.1 \quad(0.4)$ & $0.2 \quad(0.6)$ & $0.2 \quad(0.6)$ & $0.2 \quad(0.8)$ & $0.3 \quad(0.9)$ & $0.3 \quad(0.9)$ \\
\hline Mental health outpatient visits & $6.0(23.4)$ & $1.3 \quad(2.1)$ & $3.2(12.9)$ & $2.3 \quad(4.7)$ & $4.4 \quad(18.2)$ & $4.4 \quad(9.1)$ & $4.8(20.5)$ \\
\hline Nonmental health outpatient visits & $10.2(14.4)$ & $6.7 \quad(7.2)$ & $8.5 \quad(10.9)$ & $9.4 \quad(11.2)$ & $11.1 \quad(13.1)$ & $11.3(14.4)$ & $9.7 \quad(15.4)$ \\
\hline Psychotropic medications ${ }^{b}$ & $1.5 \quad(0.8)$ & $1.4 \quad(0.8)$ & $1.5 \quad(0.7)$ & $1.3 \quad(0.6)$ & $1.5 \quad(0.9)$ & $1.8 \quad(1.0)$ & $1.7 \quad(1.0)$ \\
\hline
\end{tabular}

Note: Cell values are mean (SD), unless otherwise stated.

aP $<0.05$.

${ }^{b} P<0.01$ for comparison across all antidepressant agents by chi-square tests for categorical variables or analysis of variance for count variables.

cBased on ICD-9-CM E codes.

ICD-9-CM = International Classification of Diseases, Ninth Revision, Clinical Modification; PTSD=post-traumatic stress disorder; SD=standard deviation;

SSRI = selective serotonin reuptake inhibitor.

antidepressants, those with higher numbers of psychiatric medication in the prior year were more likely to be prescribed non-SSRIs than sertraline and specifically more likely to be prescribed mirtazapine $(\mathrm{RRR}=2.35)$ or venlafaxine $(\mathrm{RRR}=2.27)$ after adjusting for other predictors. After adjusting for administrative data-based variables, we found mirtazapine more likely to be prescribed in those noted for sleep problems $(\mathrm{RRR}=2.47)$ or marital issues (RRR=2.64) than sertraline or most other antidepressants. Other than sleep problems and marital issues, we found no other chart-based variables to be significantly associated with selecting different antidepressants, statistically or by the magnitude of the association. When the chart-based variables not included in the final model presented in Table 4 were individually added to the final model, none of the dropped variables were statistically significant or had an RRR large in magnitude. The RRRs of the chart-based predictors not included in the final model ranged from 0.4 to 1.8, confirming they were not strong predictors of antidepressant selection.

\section{Discussion}

In this study, we abstracted electronic medical chart progress notes to identify factors predictive of different antidepressant treatments over and above demographic and other variables that are more easily available in administrative databases among patients who had received treatment for depression.
Administrative data typically include information needed for billing and record keeping, such as demographic variables, date and length of the visit, and ICD-9-CM diagnoses. In contrast, the medical record progress notes include more detailed information on symptoms and functioning that are relevant to clinical care. We abstracted variables reflecting the most common factors shown to influence antidepressant selection based on surveying prescribing psychiatrists, which include the avoidance of specific side effects, the presence of comorbid psychiatric disorders, and the presence of specific clinical symptoms. We found mirtazapine more likely to be prescribed in those noted for sleep problems or marital issues than sertraline or most other antidepressants. We did not find any other chart-based variables that we considered to have strong association with the selection of antidepressants after adjusting for the administrative data-based variables.

Confounding by treatment indication is a bias in observational studies of drug effects where the allocation of treatment is not randomized, resulting in an underlying heterogeneous risk profile across patients receiving different treatments. In studies of response to different antidepressant treatments, potential treatment indication bias is often a major concern where treatment indication may be related to the risk of future outcomes. Understanding factors associated with different antidepressant prescribing patterns will inform the analysis and 
TABLE 3 Chart Notations: Mental Health-Related Symptoms, Substance Use, Stressful Life Events, Suicidality, and Other Characteristics During 12 Months Prior to Newly Starting Antidepressant

\begin{tabular}{|c|c|c|c|c|c|c|c|}
\hline \multirow[b]{3}{*}{ Characteristics } & \multicolumn{4}{|c|}{ SSRI } & \multicolumn{3}{|c|}{ Non-SSRI } \\
\hline & Citalopram & Fluoxetine & Paroxetine & Sertraline & Bupropion & Mirtazapine & Venlafaxine \\
\hline & $\mathrm{n}=78$ & $\mathrm{n}=76$ & $\mathrm{n}=73$ & $n=64$ & $\mathrm{n}=86$ & $\mathrm{n}=98$ & $\mathrm{n}=81$ \\
\hline \multicolumn{8}{|c|}{ Mental health-related symptoms or a formal diagnosis } \\
\hline Anxiety or PTSD & 60.3 & 50.0 & 61.6 & 57.8 & 53.5 & 66.3 & 63.0 \\
\hline Depression & 92.3 & 89.5 & 87.7 & 93.8 & 90.7 & 94.9 & 91.4 \\
\hline Mania or manic behavior & 2.6 & 4.0 & 4.1 & 1.6 & 4.7 & 6.1 & 4.9 \\
\hline Psychosis & 5.1 & 5.3 & 4.1 & 6.3 & 4.7 & 9.2 & 6.2 \\
\hline Impulsivity & 7.7 & 2.6 & 2.7 & 7.8 & 2.3 & 7.1 & 8.6 \\
\hline Chronic or acute pain & 64.1 & 63.2 & 64.4 & 67.2 & 73.3 & 69.4 & 76.5 \\
\hline Various sleep problems ${ }^{\mathrm{a}}$ & 73.1 & 59.2 & 52.1 & 62.5 & 65.1 & 82.7 & 64.2 \\
\hline Aggression/aggressive actions & 5.1 & 1.3 & 2.7 & 4.7 & 4.7 & 5.1 & 1.2 \\
\hline \multicolumn{8}{|l|}{ Substance abuse/dependence } \\
\hline Alcohol & 21.8 & 11.8 & 19.2 & 25.0 & 18.6 & 28.6 & 19.8 \\
\hline Illicit drug & 7.7 & 7.9 & 6.9 & 14.1 & 7.0 & 15.3 & 9.9 \\
\hline Prescription drug misuse & 5.1 & 1.3 & 6.9 & 4.7 & 4.7 & 8.2 & 3.7 \\
\hline Tobacco use $\mathrm{u}^{\mathrm{a}}$ & 30.8 & 30.3 & 35.6 & 42.2 & 65.1 & 52.0 & 37.0 \\
\hline \multicolumn{8}{|l|}{ Stressful life events } \\
\hline General family problems & 23.1 & 18.4 & 17.8 & 12.5 & 16.3 & 24.5 & 18.5 \\
\hline Suicide of family member & 3.9 & 7.9 & 1.4 & 4.7 & 2.3 & 4.1 & 3.7 \\
\hline Death of family member & 11.5 & 5.3 & 15.1 & 7.8 & 7.0 & 10.2 & 7.4 \\
\hline Marital/intimate problem ${ }^{\mathrm{a}}$ & 16.7 & 10.5 & 8.2 & 18.8 & 18.6 & 34.7 & 17.3 \\
\hline Social environment problems ${ }^{\mathrm{a}}$ & 9.0 & 5.3 & 5.5 & 7.8 & 7.0 & 21.4 & 12.4 \\
\hline Difficulty access to health care & 6.4 & 7.9 & 5.5 & 1.6 & 3.5 & 8.2 & 9.9 \\
\hline Legal system/crime problems & 7.7 & 7.9 & 9.6 & 10.9 & 10.5 & 20.4 & 7.4 \\
\hline Other psychosocial or environment problems & 2.6 & 2.6 & 5.5 & 0 & 1.2 & 1.0 & 0 \\
\hline Chronic illness or pain & 82.1 & 75.0 & 72.6 & 85.9 & 79.1 & 75.5 & 81.5 \\
\hline Economic/financial problems & 28.2 & 14.5 & 21.9 & 23.4 & 27.9 & 34.7 & 28.4 \\
\hline Educational problems & 1.3 & 2.6 & 0 & 0 & 1.2 & 1.0 & 3.7 \\
\hline Occupational problems & 44.9 & 35.5 & 28.8 & 42.2 & 34.9 & 46.9 & 37.0 \\
\hline Housing problems & 11.5 & 10.5 & 15.1 & 14.1 & 15.1 & 20.4 & 19.8 \\
\hline Number of stressors, mean (SD, range) $^{\mathrm{a}}$ & $2.5(1.7,7)$ & $2.0(1.3,5)$ & $2.1(1.6,7)$ & $2.3(1.4,5)$ & $2.2(1.5,6)$ & $3.0(1.8,7)$ & $2.5(1.8,7)$ \\
\hline \multicolumn{8}{|l|}{ Suicidality or violence } \\
\hline Suicide attempt & 1.3 & 1.3 & 4.1 & 3.1 & 1.2 & 6.1 & 6.2 \\
\hline Suicidal ideation or attempt ${ }^{b}$ & 19.2 & 17.1 & 13.7 & 28.1 & 12.8 & 29.6 & 23.5 \\
\hline Suicidal plan & 6.4 & 4.0 & 8.2 & 9.4 & 5.8 & 11.2 & 11.1 \\
\hline Access to suicidal means & 6.4 & 2.6 & 5.5 & 6.3 & 2.3 & 9.2 & 9.9 \\
\hline Access to guns & 3.9 & 6.6 & 1.4 & 3.1 & 1.2 & 6.1 & 4.9 \\
\hline Violent intent & 9.0 & 4.0 & 5.5 & 4.7 & 7.0 & 9.2 & 2.5 \\
\hline Homicidal thought & 9.0 & 4.0 & 5.5 & 3.1 & 7.0 & 7.1 & 2.5 \\
\hline \multicolumn{8}{|l|}{ Mental health treatments received or considered } \\
\hline $\begin{array}{l}\text { Hospitalization considered for psychological } \\
\text { symptoms }\end{array}$ & 9.0 & 4.0 & 11.0 & 17.2 & 9.3 & 15.3 & 13.6 \\
\hline Attended AA or self-helpc & 7.7 & 0 & 5.5 & 9.4 & 10.5 & 9.2 & 2.5 \\
\hline Homelessness intervention & 5.1 & 2.6 & 11.0 & 6.3 & 3.5 & 11.2 & 8.6 \\
\hline VA substance abuse treatment ${ }^{\mathrm{c}}$ & 11.5 & 5.3 & 11.0 & 20.3 & 14.0 & 19.4 & 11.1 \\
\hline
\end{tabular}

Note: Cell values are percentages, unless otherwise stated.

${ }^{a} P<0.01$ for comparison across all antidepressant agents by chi-square tests for categorical variables or analysis of variance for count variables

${ }^{b} \mathrm{P}<0.05$.

${ }^{c}$ Does not include tobacco cessation program.

AA =Alcoholics Anonymous; PTSD = post-traumatic stress disorder; SD = standard deviation; SSRI= selective serotonin reuptake inhibitor; VA=Veterans Affairs 
TABLE 4 Adjusted RRRs from Multinomial Logistic Regression Model ${ }^{a}$ with Sertraline as Reference Antidepressant ( $N=556)$

\begin{tabular}{|c|c|c|c|c|c|c|}
\hline \multirow[b]{2}{*}{ Characteristics } & \multicolumn{3}{|c|}{ SSRI } & \multicolumn{3}{|c|}{ Non-SSRI } \\
\hline & Citalopram & Fluoxetine & Paroxetine & Bupropion & Mirtazapine & Venlafaxine \\
\hline \multicolumn{7}{|l|}{ Administrative data-based variables } \\
\hline Male & 0.32 & 0.19 & $0.11^{\mathrm{b}}$ & 0.20 & 0.50 & 0.27 \\
\hline Black (ref: nonblack race) & 0.72 & 1.07 & 2.66 & 1.11 & 2.34 & 1.36 \\
\hline Hispanic & 2.74 & 0.44 & 1.12 & 1.08 & 3.72 & 1.39 \\
\hline Age & 0.99 & $0.97 \mathrm{~b}$ & 1.00 & $0.94 \mathrm{c}$ & 1.00 & 0.99 \\
\hline Alcohol abuse/dependence & 1.27 & 0.45 & 0.74 & 0.62 & 1.75 & 0.74 \\
\hline Illicit drug abuse/dependence & 0.32 & 0.49 & 0.65 & $0.24 \mathrm{~b}$ & $0.22^{\mathrm{b}}$ & 0.50 \\
\hline Tobacco use diagnosis & 1.26 & 0.83 & 1.17 & 1.96 & 0.47 & 1.17 \\
\hline Other anxiety diagnosis & 0.69 & 0.73 & 1.45 & 0.58 & 0.58 & 0.41 \\
\hline Charlson index = $1($ ref: index $=0)$ & 1.78 & 1.43 & 1.10 & 1.90 & 0.49 & 0.42 \\
\hline Charlson index $>1$ (ref: index $=0)$ & 1.41 & 1.81 & 1.17 & 1.79 & 0.81 & 0.92 \\
\hline Number of psychiatric medications & 1.51 & 1.35 & 1.32 & 1.46 & $2.35^{\mathrm{c}}$ & $2.27 \mathrm{c}$ \\
\hline Years since depression diagnosis & 1.25 & 1.08 & 1.08 & 1.64 & 1.58 & 1.63 \\
\hline \multicolumn{7}{|l|}{ Chart-based variables } \\
\hline Tobacco use notated & 0.52 & 0.66 & 0.84 & 1.69 & 1.40 & 0.70 \\
\hline Sleep problems & 1.85 & 0.97 & 0.62 & 1.08 & $2.47^{\mathrm{b}}$ & 1.03 \\
\hline Marital/intimate problems & 1.02 & 0.55 & 0.48 & 0.66 & $2.64 \mathrm{~b}$ & 1.08 \\
\hline \multicolumn{7}{|c|}{$\begin{array}{l}\text { aThe model only included variables that are either statistically significant in antidepressant selection or presumed to be clinically significant for consideration of } \\
\text { antidepressant selection. } \\
b P<0.05 \text {. }\end{array}$} \\
\hline
\end{tabular}

interpretation of observational studies designed to compare treatment effects or health risks or to examine determinants of differential responses to the different antidepressants. Our goal was to see if further insights can be gained from measures available from medical records data regarding whether confounding by indication might explain any associations suggested or shown between different antidepressant agents and various health outcomes, including suicide. Such findings would also suggest that future observational studies can benefit greatly from spending extra effort to obtain measures available in charts to further adjust for heterogeneous risk profiles, particularly in designing pharmacoepidemiological studies.

The chart-based variables further explained variability in antidepressant selection than administrative data-based variables alone. We found patients with sleep problems and those noted with marital or intimacy problems to be more likely to be prescribed mirtazapine than sertraline. However, despite the extensive list of chart-based variables that we have considered in this analysis, we did not find any to be highly predictive of antidepressant selection. Our prior studies comparing suicide risk associated with antidepressants suggested that sertraline and fluoxetine have lower suicide risk than citalopram or paroxetine, after adjusting for administrative data-based variables using several alternate analytic approaches, although the results were not conclusive. ${ }^{6}$ A particular interest in the current study, therefore, was to assess if chart-based variables suggest any differential treatment indication. For example, if the chartbased variables measuring suicidality were shown to be negatively associated with sertraline or fluoxetine treatment, then treatment indication bias might have explained the potentially lower suicide risk associated with sertraline or fluoxetine. After adjusting for administrative data-based variables, however, we did not find many chart-based variables to be associated with selecting a particular SSRI.

Despite a relatively small sample size $(\mathrm{N}=556)$, the directions of the association between administrative data-based variables and antidepressant selection found in this study are consistent with our previous study, which assessed predictors of alternative antidepressant agent initiation based purely on administrative data $(\mathrm{N}=502,179) .{ }^{8}$ In addition, the chart-based variables found to be associated with antidepressant selection in this study are consistent with what has been generally known to this day. For example, research has shown that mirtazapine, taken at bedtime, has a positive impact on insomnia symptoms, to improve the percentage of time in bed spent asleep, decreases nighttime awakenings, and increases total time spent asleep. ${ }^{13,14}$ Unlike SSRIs, mirtazapine is also known not to have sexual side effects, which may be consistent with our finding that it is prescribed for those with marital or intimacy issues. ${ }^{15-17}$ The consistency of our results with other studies gives credence to the lack of finding many significant chart-based predictors in this study. 


\section{Limitations}

Our study is based on VHA users, a primarily male and older patient population, and thus reflects the demographic and psychosocial characteristics of the VHA patient population and has limitations in generalizability. We have only included patients who initiated an antidepressant after 12 months of no antidepressant fills. To the extent that prior treatment history, including prior positive or failed response to an antidepressant, is a factor influencing medication choice, our study did not consider antidepressant experience more than 12 months prior to the start of the index antidepressant as a potential factor. Our study also did not include weight concerns as factors influencing antidepressant selection. We did not consider discontinuation syndrome or drug-drug interactions; however, they are considered to rarely influence antidepressant selection. ${ }^{18}$

\section{Conclusions}

Our results suggest that although chart-based variables can provide predictors of antidepressant selection, they do not show notably differential treatment indication across the SSRIs, and the strength of the associations do not show highly significant differences in severity across all 7 antidepressants considered here. Our study suggests that studies comparing antidepressant agents may not benefit greatly by further adjusting for risks associated with outcomes based on variables obtained from medical records data beyond risk adjustment by administrative data-based variables.

\section{Authors}

HYUNGJIN MYRA KIM, ScD, is Research Investigator, VA Center for Clinical Management Research, VA Ann Arbor Healthcare System, and Research Scientist, Center for Statistical Consultation and Research, University of Michigan, Ann Arbor. KARA ZIVIN, $\mathrm{PhD}$, is Research Investigator, VA Center for Clinical Management Research, VA Ann Arbor Healthcare System, and Associate Professor, Department of Psychiatry, University of Michigan Medical School, Ann Arbor. HAE MI CHOE, PharmD, is Associate Professor, Department of Clinical, Social, and Administrative Sciences, University of Michigan College of Pharmacy, and CLARE M. STANO, MA, is Research Associate, Department of Psychiatry, University of Michigan Medical School, Ann Arbor. DARA GANOCZY, MPH, is Research Health Science Specialist, VA Center for Clinical Management Research, VA Ann Arbor Healthcare System, and HEATHER WALTERS, MS, is Health Science Specialist, VA Center for Clinical Management Research, VA Ann Arbor Healthcare System, and Research Project Manager, Department of Psychiatry, University of Michigan Medical School, Ann Arbor. MARCIA VALENSTEIN, MD, is Research Investigator, VA Center for Clinical Management Research, VA Ann Arbor Healthcare System, and Professor, Department of Psychiatry, University of Michigan Medical School, Ann Arbor.

AUTHOR CORRESPONDENCE: Hyungjin Myra Kim, ScD, Center for Statistical Consultation and Research, University of Michigan, 3550 Rackham, Ann Arbor, MI 48109-1070. Tel.: 734.647.8158; Fax: 734.647.2440; E-mail: myrakim@umich.edu.

\section{DISCLOSURES}

The authors declare no conflicts of interest. This study was supported by an NIH grant (R01 MH 078698).

Study concept and design were contributed by Kim and Valenstein. Stano was responsible for data collection. Data analysis was performed by Kim, Valenstein, and Stano. The manuscript was primarily written by Kim, with help from Valenstein and the other authors, and revised by Zivin, Choe, Valenstein, and Kim, assisted by Stano, Ganoczy, and Walters.

\section{REFERENCES}

1. Garrison GD, Levin GM. Factors affecting prescribing of the newer antidepressants. Ann Pharmacother. 2000;34(1):10-14.

2. Glassman AH, O'Connor CM, Califf RM, et al.; Sertraline Antidepressant Heart Attack Randomized Trial (SADHEART) Group. Sertraline treatment of major depression in patients with acute MI or unstable angina. JAMA. 2002;288(6):701-09.

3. Honig A, Kuyper AM, Schene AH, et al.; MIND-IT investigators. Treatment of post-myocardial infarction depressive disorder: a randomized, placebocontrolled trial with mirtazapine. Psychosom Med. 2007;69(7):606-13.

4. Healy D, Aldred G. Antidepressant drug use \& the risk of suicide. Int Rev Psychiatry. 2005;17(3):163-72.

5. Jick H, Kaye JA, Jick SS. Antidepressants and the risk of suicidal behaviors. JAMA. 2004;292(3):338-43.

6. Valenstein M, Kim HM, Ganoczy D, et al. Antidepressant agents and suicide death among US Department of Veterans Affairs patients in depression treatment. J Clin Psychopharmacol. 2012;32(3):346-53.

7. Charlson ME, Pompei P, Ales KL, MacKenzie CR. A new method of classifying prognostic comorbidity in longitudinal studies: development and validation. J Chron Dis. 1987;40(5):373-83.

8. Kim HM, Zivin K, Ganoczy D, et al. Predictors of alternative antidepressant agent initiation among U. S. veterans diagnosed with depression. Pharmacoepidemiol Drug Saf. 2010;19(10):1049-56.

9. Seyfried L, Hanauer DA, Nease D, Albeiruti R, Kavanagh J, Kales HC. Enhanced identification of eligibility for depression research using an electronic medical record search engine. Int J Med Inform. 2009;78(12):e13-el8

10. Kim HM, Smith EG, Stano CM, et al. Validation of key behaviourally based mental health diagnoses in administrative data: suicide attempt, alcohol abuse, illicit drug abuse and tobacco use. BMC Health Serv Res. 2012;12:18.

11. Pokorny AD. Suicide prediction revisited. Suicide Life Threat Behav. 1993;23(1):1-10

12. Platt $\mathrm{S}$, Hawton K. Suicidal behaviour and the labour market. In: Hawton K, Van Heeringen K, eds. International Handbook of Suicide and Attempted Suicide. London: John Wiley \& Sons; 2000:309-84.

13. Winokur A, Sateia MJ, Hayes JB, Bayles-Dazet W, MacDonald MM, Gary KA. Acute effects of mirtazapine on sleep continuity and sleep architecture in depressed patients: a pilot study. Biol Psychiatry. 2000;48(1):75-78. 14. Schittecatte M, Dumont F, Machowski R, Cornil C, Lavergne F, Wilmotte J. Effects of mirtazapine on sleep polygraphic variables in major depression. Neuropsychobiology. 2002;46(4):197-201.

15. Montejo-González AL, Llorca G, Izquierdo JA, et al. SSRI-induced sexual dysfunction: fluoxetine, paroxetine, sertraline, and fluvoxamine in a prospective, multicenter, and descriptive clinical study of 344 patients. J Sex Marital Ther. 1997;23(3):176-94.

16. Higgins A, Nash M, Lynch AM. Antidepressant-associated sexual dysfunction: impact, effects, and treatment. Drug Healthc Patient Saf. 2010;2:141-50.

17. Nurnberg HG, Hensley PL, Heiman JR, Croft HA, Debattista C, Paine S. Sildenafil treatment of women with antidepressant-associated sexual dysfunction: a randomized controlled trial. JAMA. 2008;300(4):395-404.

18. Zimmerman M, Posternak M, Friedman M, et al. Which factors influence psychiatrists' selection of antidepressants? Am J Psychiatry. 2004;161(7):1285-89 